\title{
Chebyshev Operational Matrix Method for Lane-Emden Problem
}

https://doi.org/10.1515/nleng-2017-0157

Received November 26, 2017; revised January 16, 2018; accepted February 21, 2018.

\begin{abstract}
In the this paper, a new modified method is proposed for solving linear and nonlinear Lane-Emden type equations using first kind Chebyshev operational matrix of differentiation. The properties of first kind Chebyshev polynomial and their shifted polynomial are first presented. These properties together with the operation matrix of differentiation of first kind Chebyshev polynomial are utilized to obtain numerical solutions of a class of linear and nonlinear LaneEmden type singular initial value problems (IVPs). The absolute error of this method is graphically presented. The proposed framework is different from other numerical methods and can be used in differential equations of the same type. Several examples are illuminated to reveal the accuracy and validity of the proposed method.
\end{abstract}

Keywords: Lane-Emden equations, chebyshev first kind operational matrix of differentiation, Isothermal gas spheres equation, Nonlinear ODE

\section{Introduction}

In astrophysics, Lane-Emden equation model is one of the case of singular initial value problem in the form of second-order nonlinear ordinary differential equation(ODE), which describes several important phenomenon in mathematical science and astrophysics such as radiative cooling, behavior of spherical self-gravitating gas clouds, stellar structure [1], thermal explosions [2],

Bhuvnesh Sharma, M.K. Paswan, Department of Mechanical Engg., National Institute of Technology, Jamshedpur-831014, Jharkhand, India

*Corresponding Author: Sunil Kumar, Department of Mathematics, National Institute of Technology, Jamshedpur-831014, Jharkhand, India, E-mail: skiitbhu28@gmail.com, skumar.math@nitjsr.ac.in Dindayal Mahato, Department of Computer Science and Engg., National Institute of Technology, Jamshedpur-831014, Jharkhand, India modeling of cluster of galaxies, treatment of a phase transition in critical absorption, thermionic currents [3]. The dimensionless Poisson's equation which describes the equilibrium density distribution in self-gravitating symmetric sphere of polytropic isothermal gas, was proposed by Jonathan H. \& Lane [4]. The further equation described by Robert \& Emden [5] which is known as Lane-Emden equation. This equation dictates the pressure and density variation with respect to each other, formulated as

$$
y^{\prime \prime}(x)+\frac{\alpha}{x} y^{\prime}(x)+f(x, y)=g(x) \quad \alpha, x \geq 0,
$$

subject to supplementary conditions:

$$
y(0)=A, \quad y^{\prime}(0)=0
$$

where $A$ is constant, $f(x, y)$ is a continuous real valued function, and $g(x) \in C[0,1]$. It is known that the analytical solution of lane-Emden equation exists for supplementary conditions(2) in the locality of the singular point at $x=0$. Taking $\alpha=2, g(x)=0, f(x, y)=y^{n}$ and $A=1$ in equation (1) and (2) respectively

$$
y^{\prime \prime}(x)+\frac{2}{x} y^{\prime}(x)+y^{n}=0
$$

characteristically, Eq. (3), represents the standard LaneEmden equation, which will be subjected to the supplementary conditions as given in Eq. (4).

$$
y(0)=1, \quad y^{\prime}(0)=0
$$

Similarly, Isothermal gas spheres are modeled by Davis [6]

$$
y^{\prime \prime}(x)+\frac{2}{x} y^{\prime}(x)+e^{y}=0
$$

subject to supplementary conditions:

$$
y(0)=0, \quad y^{\prime}(0)=0
$$

Various methods have been presented to solve the LaneEmden equation in unbound domain. Wazwaz et al. [7] presented the series solution of integral form of Lane-Emden equation by using Adomian Decomposition method. Bender et al. [8] proposed a new perturbative technique by replacing nonlinear terms in the Lagrangian and introduced a new parameter ?. Aminikhah et al. [9] presented the numerical solution of Lane-Emden equation 
based on cubic B-spline approximation. Krivec and Mandelzweig $[10,11]$ proposed quasilinearization approach to solve the Lane-Emden equation with singular potentials. Ramos [12] introduced the linearization methods for the piece-wise closed form solution of Lane-Emden equation, which depends on the Jacobian and derivative of independent variable. The other effective methods are variation iterative methods [13], non-perturbative approximation method [14] and the Pade-series method [15]. Russell and Shampine [16] explored the Lane-Emden equation for linear function $f(x, y)=k y+h(x)$ and proved that a unique solution exists if $h(x) \in C[0,1]$ and $-\infty<k \leq \pi^{2}$.

Hermite, Laguerre, Legendre and Chebyshev polynomials are orthogonal over unbounded domain. These polynomial spectral methods are most common methods due to reveal the more accurate solution of a class of LaneEmden equation type [17-20]. Parand et al. [21] proposed Hermite collocation method for Lane-Emden equation. Pandey et al. [22] developed efficient numerical method for solving Lane-Emden equation using Legendre operation matrix of differentiation. Doha et al. [23] presented a new algorithm using second kind chebyshev operational matrix of derivative to obtain numerical solutions of LaneEmden type IVPs. Abd-Elhameed et al. [24, 25] proposed a new algorithm to describe Lane-Emden Singular type IVPs using new Galerkin operation matrix of differentiation.

Wavelet theory is a relatively new and comes into view of mathematical research area. It has been applied with the orthogonal polynomials to propose a new method for solving several engineering problems. Yousefi [26] has given the solution of Lane-Emden equation using Legendre wavelet method. Youssri et al. [27] introduced ultraspherical wavelets operational matrix of derivatives for approximation of a class of Lane-Emden type with high accuracy.

The aim of this paper is to develop a more accurate and fast computational method for solving linear and nonlinear Lane-Emden equation (1) using first kind Chebyshev operation matrix of differentiation. The manuscript is organized as follows: Section 2 introduces the definition of first kind Chebyshev polynomial and formulates differential operation matrix. Section 3 describes the application of differentiation matrix of Chebyshev polynomial for solving the Lane-Emden equation. Section 4 exhibits the approximate solution obtained by the proposed method for the case of physical significance of polytropic index range $0<n \leq 5$, wherein the result shows high accuracy and efficiency than other existing numerical solutions.

\section{First kind chebyshev polynomials and its operational matrix of Differentiation}

The analytic form of the shifted chebyshev polynomial $P_{m}(x)$ of degree $m$ are given by Atabakzadeh et al. [28]

$P_{m}(x)=\sum_{k=0}^{\left[\frac{m}{2}\right]}(-1)^{k} 2^{m-2 k-1} \frac{m}{m-k}\left(\begin{array}{c}m-k \\ k\end{array}\right)(2 x-1)^{m-2 k}$

Any function, $y(x) \in L^{2}[0,1]$ can be approximated as a sum of shifted chebyshev polynomial (of the first kind) as:

$$
y(x)=\sum_{i=0}^{\infty} c_{i} P_{i}(x)
$$

where

$c_{i}=\left\langle y(x), P_{i}(x)\right\rangle=(2 i+1) \int_{0}^{1} y(x) P_{i}(x) d x, \quad i=0,1, \cdots$

In general, the series in Equation(8) can be truncated with the first $(N+1)$ shifted chebyshev polynomial of first kind as

$$
y_{N}(x)=\sum_{i=0}^{N} c_{i} P_{i}(x)=C^{T} \phi(x)
$$

where

$C^{T}=\left[\begin{array}{llll}c_{0} & c_{1} & \cdots & c_{N}\end{array}\right], \quad \phi(x)=\left[\begin{array}{lllll}P_{0}(x) & P_{1}(x) & \cdots & P_{N}(x)\end{array}\right]^{T}$

The operation matrix of derivative of the shifted chebyshev polynomial set $\phi(x)$ is defined as

$$
\frac{d \phi(x)}{d x}=D^{(1)} \phi(x)
$$

where $D^{(1)}$ in the $(N+1) \times(N+1)$ operation matrix of differentiation of chebyshev polynomial given as:

$$
\begin{aligned}
& D^{(1)}=d_{i j}=
\end{aligned}
$$

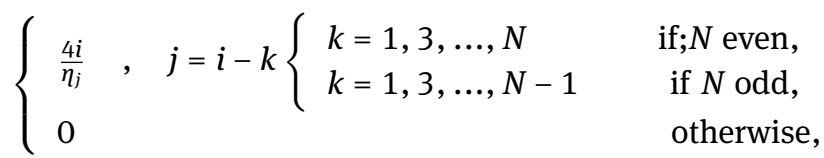

where $\eta_{0}=2$ and $\eta_{k}=1(k \geq 1)$. 
For example $N=6$, we have

$$
D^{(1)}=2\left(\begin{array}{cccccc}
0 & 0 & 0 & 0 & 0 & 0 \\
1 & 0 & 0 & 0 & 0 & 0 \\
0 & 4 & 0 & 0 & 0 & 0 \\
3 & 0 & 6 & 0 & 0 & 0 \\
0 & 8 & 0 & 8 & 0 & 0 \\
5 & 0 & 10 & 0 & 10 & 0
\end{array}\right)
$$

where $m=0,1,2 \cdots N$.

From Eq. (10), it can be generalized for any $n \in N$ as: $\frac{d^{n} \phi(x)}{d x}=\left(D^{(1)}\right)^{n} \phi(x)=D^{n} \phi(x)$, where $n=1,2,3, \cdots$

\section{Application of the method}

This section describe the application of differentiation matrix of chebyshev polynomial for solving the Lane-Emden equation. The Lane-Emden equation is formulated as

$$
y^{\prime \prime}(x)+\frac{\alpha}{x} y^{\prime}(x)+f(x, y)=g(x) \quad \alpha, x \geq 0,
$$

subject to supplementary conditions: $y(0)=A, y^{\prime}(0)=0$. Approximating $y(x), f(x, y)$ and $g(x)$ by the shifted chebyshev polynomial has

$$
\begin{gathered}
y(x) \approx \sum_{i=0}^{n} c_{i} P_{i}(x)=C^{T} \phi(x), \\
f(x, y) \approx f\left(x, C^{T} \phi(x)\right), \\
g(x) \approx \sum_{i=0}^{n} g_{i} P_{i}(x)=G^{T} \phi(x),
\end{gathered}
$$

where the unknowns are, $C=\left[\begin{array}{llll}c_{0} & c_{1} & \cdots & c_{N}\end{array}\right]^{T}$. Using first kind chebyshev polynomial operation matrix of derivative, equation (15) can be expressed as

$$
C^{T} D^{2} \phi(x)+\frac{\alpha}{x} C^{T} D^{1} \phi(x)+f\left(x, C^{T} \phi(x)\right) \approx G^{T} \phi(x)
$$

The Residual $R_{N}(x)$ for equation(18) can be expressed as

$$
R_{N}(x) \approx C^{T} D^{2} \phi(x)+\frac{\alpha}{x} C^{T} D^{1} \phi(x)+f\left(x, C^{T} \phi(x)\right)-G^{T} \phi(x)
$$

Eq. (18) is converted in $N-2$ nonlinear equations by applying Tau method [29], using following equation,

$$
\begin{aligned}
& \left\langle R_{N}(x), P_{i}(x)\right\rangle=\int_{0}^{1} R_{N}(x) P_{i}(x) d x=0, \\
& i=0,1,2, \cdots, N-2 .
\end{aligned}
$$

The supplementary conditions of Eq. (17) are given by

$$
y(0)=C^{T} \phi(0)=d_{0}, \quad y^{(1)}(0)=C^{T} D^{(1)} \phi(0)=d_{1} .
$$

By substitute the shifted polynomials in Eq. (20), we have $(N-2)$ nonlinear equations. These equation together with Eq. (24) generate $N$ nonlinear equations. This nonlinear system can be solved using Newton iterative method to determine coefficients of vector $C$. We can get the numerical solution $y(x)$ of Lane-Emden equation by substituting $C$ into Eq. (15).

\section{Convergence and error analysis}

In this section a convergence analysis is given for the method of solution discussed in last section for linear system of differential equations. It is well-known that shifted Chebyshev polynomials $\tau_{i}(x)$ forms a complete orthogonal set [30], $\Omega=[0,1]$ and $L_{\omega}^{2}(\Omega)$ denotes the space of all functions $u: \Omega \rightarrow \mathbb{R}$ with weighted $L^{2}$-norm and defined by

$$
\|u\|_{L_{\omega}^{2}(\Omega)}=\int_{0}^{1} u^{2}(t) \omega(t) d t
$$

we recall that $H_{\omega}(\Omega)$ is the sobolev space of all function $u(t)$ on $\Omega$ such that $u(t)$ and all its weak derivative up to order $m$ are in $L_{\omega}$ and define $\|\cdot\|_{H_{\omega}^{l, N}}(\Omega)$ as

$$
\|u\|_{H_{\omega}^{m}(\Omega)}=\left(\sum_{k=0}^{m}\left\|\frac{\partial^{k}}{\partial t^{k}} u(t)\right\|_{L_{\omega}^{2}(\Omega)}^{2}\right)^{\frac{1}{2}}
$$

The semi-norm also defined by

$$
|u|_{H_{\omega}^{m, N}(\Omega)}=\sum_{i=\min (m, N)}^{N} N\left\|u^{(i)}(t)\right\|_{L_{\omega}^{2}(\Omega)}^{2}
$$

Now, suppose $u_{N}=\sum_{k=0}^{N} \hat{U}_{k} \tau_{k}$ is the truncated Chebyshev approximations of a function $u \in H_{\omega}^{m}(\Omega)$. Then, as has been proved in [30], the truncation error is

$$
\| u-\left.u_{N}\right|_{H_{\omega}^{l, N}(\Omega)} \leq C N^{2 l-l / 2-m}|u|_{H_{\omega}^{m, N}}(\Omega)
$$

For any $1 \leq l \leq m$ and $C$ is a positive constant independent of $m$. Then, using proposed analytical approximation method, the approximation of $y$ is $y_{N}=$ $\left(y_{1 N}, y_{1 N}, \ldots, y_{n N}\right), y_{i N}(x)=\sum_{k=0}^{N} c_{i k} \tau_{k}(x)$ and error is defined by $e(x)=\left(e_{1}, e_{2}, \ldots, e_{n}\right)=y(x)=y_{N}(x)$. Therefore,

$$
D^{2} e_{i}+\sum_{j=1}^{k_{i}} a_{i j} e_{j}=e_{g i}, \quad i=1,2, \ldots, n,
$$


with $e_{g i}=g_{i}-g_{i N}$ and $g_{i k} \tau_{k}(x)$. Taking the norm $\|\cdot\|_{H_{\omega}^{l, N}(\Omega)}$ from both sides of (23) yields

$$
\begin{aligned}
\left\|e_{g i}\right\|_{H_{\omega}^{l, N}(\Omega)}= & \left\|D^{2} e_{i}+\sum_{j=1}^{k_{i}} a_{i, j} e_{j}\right\|_{H_{\omega}^{l, N}(\Omega)} \leq\left\|D^{2} e_{i}\right\| \\
& +\sum_{j=1}^{k_{i}}\left|a_{i, j}\right|\left\|e_{j}\right\|_{H_{\omega}^{l, N}(\Omega)} .
\end{aligned}
$$

On the other hand, since operator $D^{2}$ on Chebyshev polynomial space is continuous and bounded, as has been proved in [31], there exists a constant $\bar{C}$ such that $\left\|D^{2} e_{i}\right\|_{H_{\omega}^{l, N}(\Omega)} \leq \bar{C}\left\|e_{i}\right\|_{H_{\omega}^{l, N}(\Omega)}$. Now, using this and Eq. (24) we find

$$
\left\|e_{g i}\right\|_{H_{\omega}^{l, N}(\Omega)} \leq \bar{C}\left\|e_{i}\right\|_{H_{\omega}^{l, N}(\Omega)}+\sum_{j=1}^{k_{i}}\left|a_{i, j}\right||| e_{j} \|_{H_{\omega}^{l, N}(\Omega)} .
$$

Let the vector norm $\|e\|_{H_{\omega}^{l, N}(\Omega)}=\max _{1 \leq i \leq n}\left\|e_{i}\right\|_{H_{\omega}^{l, N}(\Omega)}$ and the vector semi-norm $\left.|y|_{H_{\omega}^{l, N}(\Omega)} \max _{1 \leq i \leq n}|| y_{i}\right|_{H_{\omega}^{l, N}(\Omega)}$. Then Eq. (25) yields

$$
\begin{aligned}
\left\|e_{g i}\right\|_{H_{\omega}^{l, N}(\Omega)} & \leq \bar{C}\|e\|_{H_{\omega}^{l, N}(\Omega)}+\sum_{j=1}^{k_{i}}\left|a_{i, j}\right|\|e\|_{H_{\omega}^{l, N}(\Omega)} \\
& \leq\|e\|_{H_{\omega}^{l, N}(\Omega)}\left(\bar{C}+\sum_{j=1}^{k_{i}}\left|a_{i, j}\right|\right) .
\end{aligned}
$$

Finally, combining (22) and (26) gives the following bound for the error in our presented analytical approximation method

$$
\frac{\|\left. e_{g i}\right|_{H_{\omega}^{l, N}(\Omega)}}{\mathbb{A}} \leq \|\left. e\right|_{H_{\omega}^{l, N}(\Omega)} \leq C N^{2 l-l / 2-m}|y|_{H_{\omega}^{m, N}}(\Omega)
$$

where $\mathbb{A}=\bar{C}+\max _{1 \leq i \leq n} \sum_{j=1}^{k_{i}}\left|a_{i, j}\right|$. Note that single $\Omega$ is compact set, $|y|_{H_{\omega}^{m, N}}(\Omega)$ is bounded and this means that our approximation converges for sufficiently large $N$.

Remark 1. As we have stated above, the convergence analysis for the proposed method just applied into the linear system (20). However, convergence analysis cannot simply determine for the nonlinear case as above discussion and needs some more efforts. Indeed for each nonlinear case, we may need some additional conditions on nonlinear functions $y^{n}(x)$ in system (20).

\section{Numerical results and discussions}

In this section, the introduced first kind Chebyshev operational matrix of derivatives is employed for solving both linear ana nonlinear Lane-Emden IVPs. The singularity in the variable coefficient $(\alpha / x)$ is handled by reducing a class of Lane-Emden type singular initial value problems to a system of algebraic equations using Tau method and supplementary conditions. The Newton iterative method is used to determine the coefficients of system equations. Six examples are executed on MATLAB-2016 using the proposed numerical algorithm to show the accuracy and efficiency of Chebyshev operation matrix method.

Let $f(x, y)=y^{n}(x), g(x)=0$ and $A=1$, Eq.(1) can be written as

$$
y^{\prime \prime}(x)+\frac{2}{x} y^{\prime}(x)+y^{n}(x)=0
$$

with supplementary conditions

$$
y(0)=1, \quad y^{\prime}(0)=0
$$

Example 1. for $\mathbf{n}=\mathbf{0}$, the linear Lane-Emden equation from Eq. (27) has the form

$$
y^{\prime \prime}(x)+\frac{2}{x} y^{\prime}(x)+1=0
$$

with supplementary conditions

$$
y(0)=1, \quad y^{\prime}(0)=0
$$

Applying the method, proposed in section 3 for $N=2$, we have

$$
y(x)=C^{T} \phi(x)=c_{0} P_{0}(x)+c_{1} P_{1}(x)+c_{2} P_{2}(x)
$$

From Eq.(18), $\quad D^{(1)}=\left(\begin{array}{ccc}0 & 0 & 0 \\ 2 & 0 & 0 \\ 0 & 8 & 0\end{array}\right)$ and $D^{(2)}=$ $\left(\begin{array}{ccc}0 & 0 & 0 \\ 0 & 0 & 0 \\ 12 & 0 & 0\end{array}\right)$

By using Eq. (25), we obtain

$$
48 c_{2}+1=0,
$$

By applying the supplementary conditions from Eq. (27) we have

$$
\begin{gathered}
c_{0}-c_{1}+c_{2}=1, \\
2 c_{1}-8 c_{2}=0,
\end{gathered}
$$

Solving Eqs. (32)-(34) we get,

$$
c_{0}=\frac{15}{16}, \quad c_{1}=\frac{-1}{12}, \quad c_{2}=\frac{-1}{48} .
$$

Substituting the values of constant vector in Eq. (31), the solution is obtained as:

$$
\begin{aligned}
y(x) & =c_{0} P_{0}(x)+c_{1} P_{1}(x)+c_{2} P_{2}(x) \\
& =\left(\begin{array}{lll}
\frac{15}{16} & \frac{-1}{12} & \frac{-1}{48}
\end{array}\right)\left(\begin{array}{c}
1 \\
2 x-1 \\
8 x^{2}-8 x+1
\end{array}\right)=1-\frac{x^{2}}{6},
\end{aligned}
$$


which is the exact solution.

Example 2. For $\mathbf{n}=\mathbf{1}$, the nonlinear Lane-Emden equation from Eq. (27) has the form

$$
y^{\prime \prime}(x)+\frac{2}{x} y^{\prime}(x)+y(x)=0
$$

with supplementary conditions

$$
y(0)=1, \quad y^{\prime}(0)=0
$$

Solving Eq. (35), the exact solution is $y(x)=\frac{\sin x}{x}$. Here,

$$
f(x, y)=y(x)=C^{T} \phi(x) .
$$

The proposed method has been applied into the Eq. (35) with supplementary conditions Eq. (36) and the unknown matrix $C^{T}$ has evaluated by solving the nonlinear system for $N=8$ and $N=10$. The values of coefficients are are shown in Table 4 and 5 respectively.The exact solution and obtained solution using shifted first kind chebyshev polynomial in larger range $[0,8]$ are plotted in Fig. 1 and absolute error is graphically presented in Fig. 2.

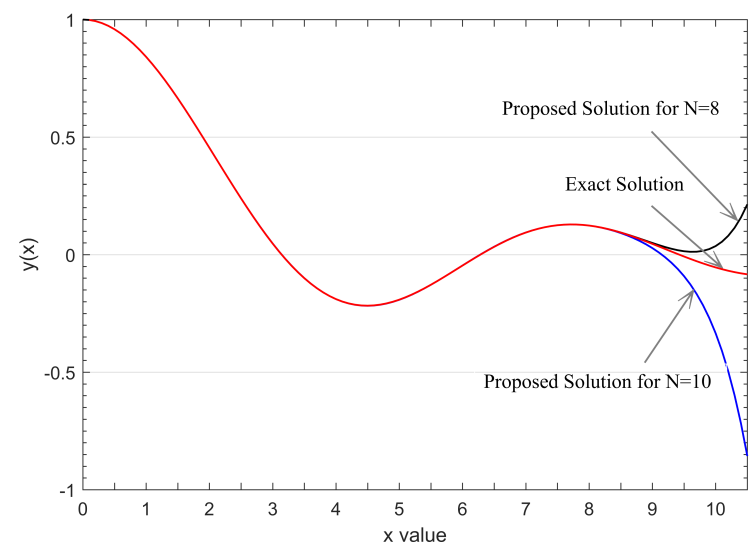

Fig. 1: Plot between $x$ \& obtained solution $y(x)$ for $N=8, N=10$ and exact solution.

Example 3. For $\mathbf{n}=\mathbf{3}$, the nonlinear Lane-Emden equation from Eq. (27) has the form

$$
y^{\prime \prime}(x)+\frac{2}{x} y^{\prime}(x)+y^{3}(x)=0,
$$

with supplementary conditions

$$
y(0)=1, \quad y^{\prime}(0)=0
$$

Here,

$$
y(x)=C^{T} \phi(x) \text { and } f(x, y)=y^{3}(x)=\left(C^{T} \phi(x)\right)^{3} .
$$

The values of unknown coefficients $C^{T}$ for $N=8$ and $N=10$ are shown in table 4 and 5 respectively. The analytic series solution [7] and obtained result using first kind
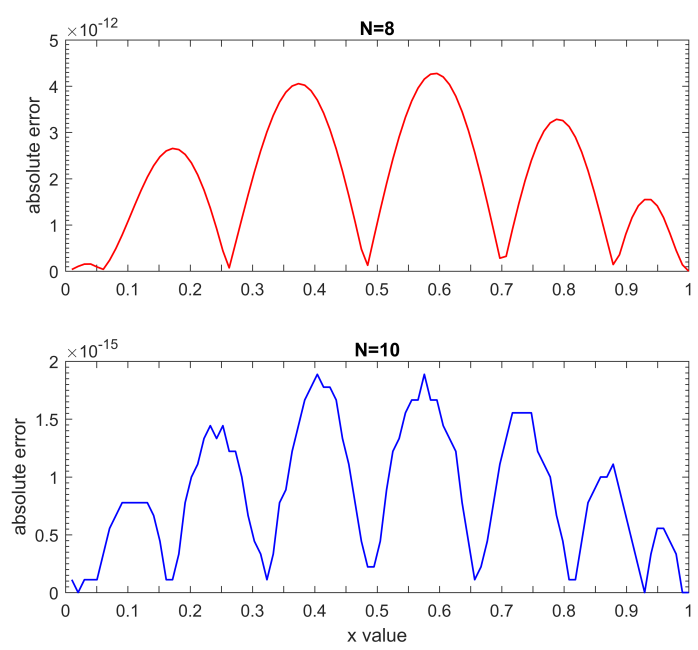

Fig. 2: Absolute errors at $N=8$ and $N=10$ for example 2 .

chebyshev polynomial are graphically presented in Fig. 3 in range $[0,5]$.

The Table 1 shows the comparison of results and absolute errors with Wazwaz et al. [7] and Pandey et al. [22]. From Table 1 and Fig. 3, we observe that the proposed method providing better results compare to the recently developed methods.

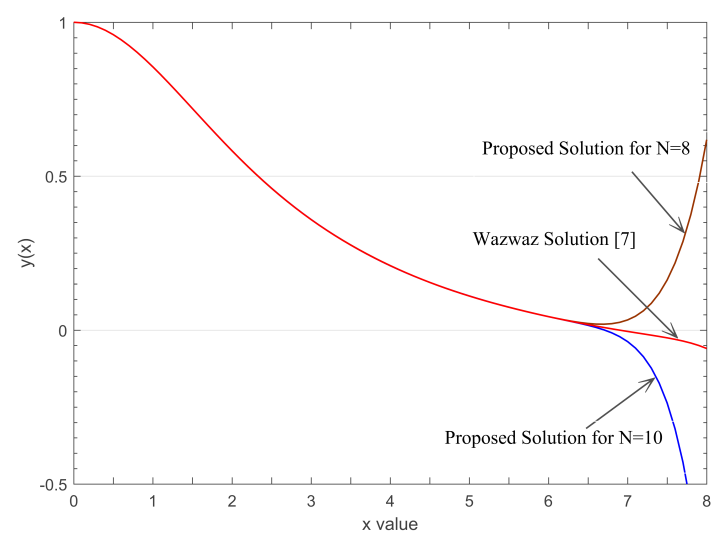

Fig. 3: Plot of obtained solution y(x) for $N=8, N=10$ and series solution presented by Wazwaz [7] for example 3 .

Example 4. For $\mathbf{n}=\mathbf{5}$, the nonlinear Lane-Emden equation from Eq. (27) has the form

$$
y^{\prime \prime}(x)+\frac{2}{x} y^{\prime}(x)+y^{5}(x)=0,
$$

with supplementary conditions

$$
y(0)=1, \quad y^{\prime}(0)=0
$$


Table 1: Comparison of approximate solutions and error obtained by modified method with series solution wazwaz [7] and newly advanced method Pandey [22] for Example 3.

\begin{tabular}{ccccc}
\hline $\mathrm{x}$ & Present method & Wazwaz [7] & Error at $N=10$ & Error at Pandey [22] \\
\hline 0 & 0.9999999999 & 1 & $2.23 \mathrm{E}-12$ & $1.14 \mathrm{E}-13$ \\
0.1 & 0.9983358295 & 0.9983358295 & $2.12 \mathrm{E}-11$ & $1.86 \mathrm{E}-11$ \\
0.2 & 0.9933730935 & 0.9933730935 & $1.38 \mathrm{E}-11$ & $1.65 \mathrm{E}-11$ \\
0.5 & 0.9598390699 & 0.9598391144 & $4.45 \mathrm{E}-08$ & $4.45 \mathrm{E}-08$ \\
1 & 0.8550575685 & 0.8550950543 & $3.74 \mathrm{E}-05$ & $3.74 \mathrm{E}-05$ \\
\hline
\end{tabular}

having exact solution $y(x)=\left(1+\frac{x^{2}}{3}\right)^{\frac{-1}{2}}$.

Here,

$$
y(x)=C^{T} \phi(x)
$$

and

$$
f(x, y)=y^{5}(x)=\left(C^{T} \phi(x)\right)^{5} .
$$

The unknown matrix $C^{T}$ has evaluated by solving the nonlinear system for $N=8$ and $N=10$. The values of coefficients are are shown in Table 4 and 5 respectively.The exact solution and obtained solution using shifted first kind chebyshev polynomial in larger range [0,5] are plotted in Fig. 4 and absolute error is graphically presented in Fig. 5 .

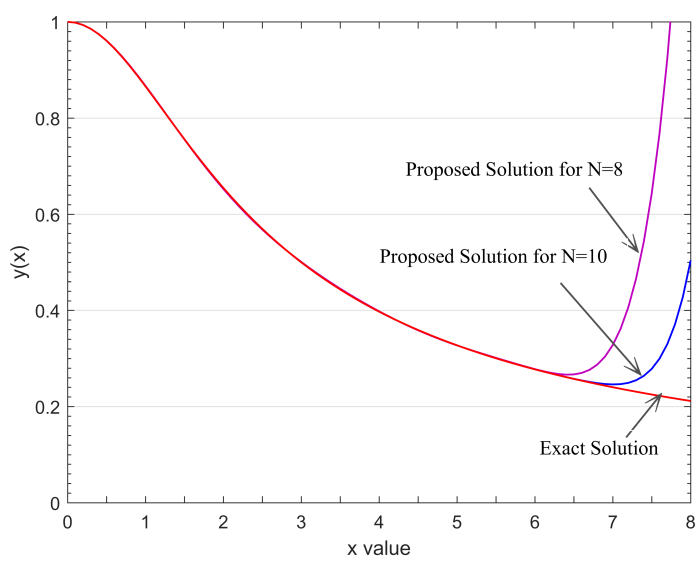

Fig. 4: Plot of obtained solution y(x) for $N=8, N=10$ and exact solution for example 4 .

Example 5. Isothermal gas spheres equation from Eq.(6) has the form

$$
y^{\prime \prime}(x)+\frac{2}{x} y^{\prime}(x)+\exp (y)=0, \quad x \geq 0,
$$

with supplementary conditions

$$
y(0)=0, \quad y^{\prime}(0)=0
$$

Here, we have
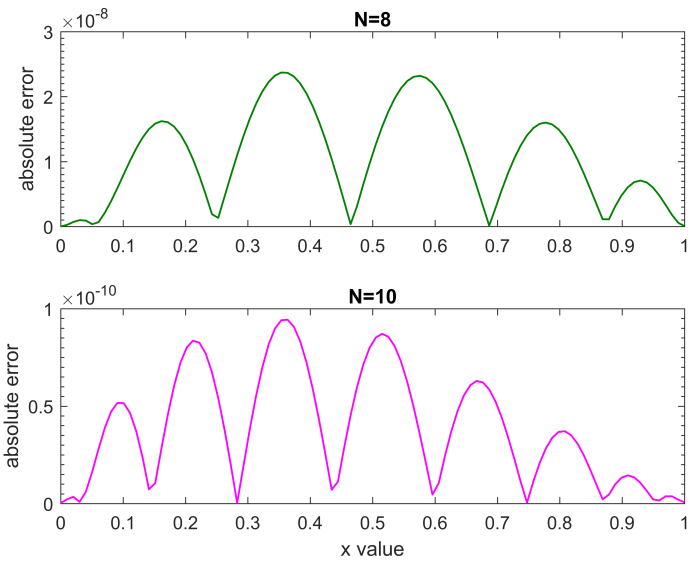

Fig. 5: Absolute errors at $N=8$ and $N=10$ for example 4 .

$$
y(x)=C^{T} \phi(x) \text { and } f(x, y)=e^{y} .
$$

Expansion of $f(x, y)$ according to Taylor series is

$$
f(x, y)=e^{y}=1+y+\frac{y^{2}}{2}+\frac{y^{3}}{6}+\frac{y^{4}}{24}+\frac{y^{5}}{120}+\cdots
$$

By considering first five terms, $f(x, y)$ can be expressed as

$$
f(x, y)=1+C^{T} \phi(x)+\frac{\left(C^{T} \phi(x)\right)^{2}}{2}+\frac{\left(C^{T} \phi(x)\right)^{3}}{6}+\frac{\left(C^{T} \phi(x)\right)^{4}}{24}
$$

The unknown matrix $C^{T}$ is shown in Tables 4 and 5 respectively. The Wazwaz [7] analytic series solution and evaluated results by solving nonlinear system using developed method for $N=8$ and $N=10$, are shown in Fig. 6 in range $[0,1]$.

The Table 2 shows the superiority of obtained approximate solutions than Wazwaz et al. [7], Pandey et al. [22] and Parand et al. [21].

Example 6. Consider the ordinary differential equation of Lane-Emden type of shape factor 2 ,

$$
y^{\prime \prime}(x)+\frac{2}{x} y^{\prime}(x)+\sin (y)=0,
$$

with supplementary conditions

$$
y(0)=1, \quad y^{\prime}(0)=0
$$


Table 2: Comparison of approximate solutions and error obtained by modified method with series solution wazwaz [7], newly advanced method Pandey [22] and Parand [21] for Example 5.

\begin{tabular}{cccccc}
\hline $\mathrm{x}$ & Present method & Wazwaz [7] & Error for $N=10$ & Error at [22] & Error at [21] \\
\hline 0 & $9.411877 \mathrm{E}-11$ & 0.0000000000 & $9.41 \mathrm{E}-11$ & $9.24 \mathrm{E}-18$ & $0.00 \mathrm{E}+00$ \\
0.1 & -0.0016658337 & -0.0016658338 & $9.93 \mathrm{E}-11$ & $5.28 \mathrm{E}-10$ & $5.85 \mathrm{E}-07$ \\
0.2 & -0.0066533669 & -0.0066533671 & $1.06 \mathrm{E}-10$ & $3.37 \mathrm{E}-08$ & $6.04 \mathrm{E}-07$ \\
0.5 & -0.0411539572 & -0.0411539567 & $3.42 \mathrm{E}-10$ & $8.12 \mathrm{E}-06$ & $5.58 \mathrm{E}-07$ \\
1 & -0.1588276829 & -0.1588272857 & $3.29 \mathrm{E}-07$ & $4.93 \mathrm{E}-04$ & $8.20 \mathrm{E}-07$ \\
\hline
\end{tabular}

Table 3: Comparison of approximate solutions and error obtained by modified method with series solution wazwaz [7], and newly advanced method Pandey [22] \& Saadtmandi and Dehgan [29] for example 6.

\begin{tabular}{cccccc}
\hline $\mathrm{x}$ & Present nethod & Wazwaz [7] & Error at $\mathrm{N}=10$ & Error at [22] & Error at [29] \\
\hline 0 & 0.9999999999 & 1.0000000000 & $3.78 \mathrm{E}-14$ & $1.11 \mathrm{E}-16$ & $0.00 \mathrm{E}+00$ \\
0.1 & 0.9985976022 & 0.9985979273 & $5.83 \mathrm{E}-08$ & $3.25 \mathrm{E}-07$ & $7.21 \mathrm{E}-06$ \\
0.2 & 0.9943949769 & 0.9943962648 & $5.42 \mathrm{E}-07$ & $1.28 \mathrm{E}-06$ & $1.00 \mathrm{E}-05$ \\
0.5 & 0.9651702487 & 0.9651777797 & $1.67 \mathrm{E}-07$ & $7.53 \mathrm{E}-06$ & $1.04 \mathrm{E}-05$ \\
1 & 0.8636571676 & 0.8636807315 & $3.27 \mathrm{E}-06$ & $2.35 \mathrm{E}-05$ & $7.03 \mathrm{E}-06$ \\
\hline
\end{tabular}

Table 4: The value of the unknowns vector $C^{T}$ for discussed examples for $N=8$.

\begin{tabular}{cccccc}
\hline coefficients & Example 2 & Example 3 & Example 4 & Example 5 & Example 6 \\
\hline$c_{0}$ & 0.9397344 & 0.9435839 & 0.9460855 & -0.0603338 & 0.9484656 \\
$c_{1}$ & -0.0797633 & -0.0736793 & -0.0686545 & -0.0798799 & -0.0684238 \\
$c_{2}$ & -0.0190577 & -0.0161548 & -0.0138788 & -0.0191298 & -0.0166709 \\
$c_{3}$ & 0.0004999 & 0.0012224 & 0.0016977 & 0.0004686 & 0.0002518 \\
$c_{4}$ & 0.0000588 & 0.0001001 & 0.0000853 & 0.0000499 & 0.0000338 \\
$c_{5}$ & -0.0000012 & -0.0000145 & -0.0000309 & $-2.536 \mathrm{E}-07$ & $6.782 \mathrm{E}-07$ \\
$c_{6}$ & $8.702 \mathrm{E}-08$ & $-3.823 \mathrm{E}-07$ & $7.421 \mathrm{E}-07$ & $-1.444 \mathrm{E}-07$ & $3.324 \mathrm{E}-08$ \\
$c_{7}$ & $1.289 \mathrm{E}-09$ & $1.398 \mathrm{E}-07$ & $4.318 \mathrm{E}-07$ & $1.293 \mathrm{E}-08$ & $-5.028 \mathrm{E}-09$ \\
$c_{8}$ & $7.557 \mathrm{E}-11$ & $9.082 \mathrm{E}-10$ & $3.783 \mathrm{E}-08$ & $3.846 \mathrm{E}-10$ & $-2.986 \mathrm{E}-10$ \\
\hline
\end{tabular}

Table 5: The value of the unknowns vector $C^{T}$ for discussed examples for $N=10$.

\begin{tabular}{cccccc}
\hline coefficients & Example 2 & Example 3 & Example 4 & Example 5 & Example 6 \\
\hline$c_{0}$ & 0.9397344 & 0.9435839 & 0.9468055 & -0.0603338 & 0.9484656 \\
$c_{1}$ & -0.0797633 & -0.0736793 & -0.0686545 & -0.0798799 & -0.0684238 \\
$c_{2}$ & -0.0190577 & -0.0161548 & -0.0138788 & -0.0191298 & -0.0166709 \\
$c_{3}$ & 0.0004999 & 0.0012224 & 0.0016977 & 0.0004686 & 0.0002518 \\
$c_{4}$ & 0.0000588 & 0.0001001 & 0.0000853 & 0.0000499 & 0.0000338 \\
$c_{5}$ & -0.0000012 & -0.0000145 & -0.0000309 & $-2.536 \mathrm{E}-07$ & $6.782 \mathrm{E}-07$ \\
$c_{6}$ & $8.702 \mathrm{E}-08$ & $-3.812 \mathrm{E}-07$ & $4.794 \mathrm{E}-07$ & $-1.444 \mathrm{E}-07$ & $3.324 \mathrm{E}-08$ \\
$c_{7}$ & $1.291 \mathrm{E}-09$ & $1.426 \mathrm{E}-07$ & $4.426 \mathrm{E}-07$ & $1.309 \mathrm{E}-08$ & $-5.04 \mathrm{E}-09$ \\
$c_{8}$ & $7.522 \mathrm{E}-11$ & $1.642 \mathrm{E}-09$ & $4.250 \mathrm{E}-08$ & $3.532 \mathrm{E}-10$ & $-2.979 \mathrm{E}-10$ \\
$c_{9}$ & $9.165 \mathrm{E}-13$ & $1.196 \mathrm{E}-09$ & $4.373 \mathrm{E}-09$ & $-6.520 \mathrm{E}-11$ & $4.790 \mathrm{E}-12$ \\
$c_{10}$ & $4.275 \mathrm{E}-14$ & $5.245 \mathrm{E}-11$ & $9.754 \mathrm{E}-10$ & $-5.20 \mathrm{E}-13$ & $6.51 \mathrm{E}-13$ \\
\hline
\end{tabular}




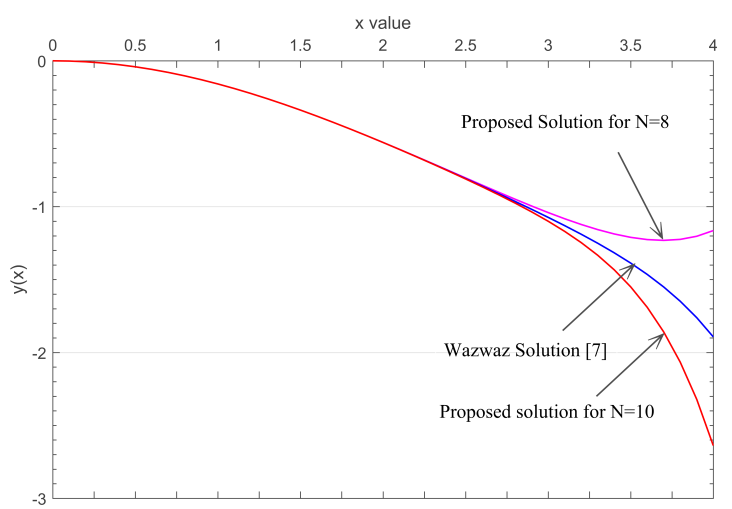

Fig. 6: Plot of obtained solution $\mathrm{y}(\mathrm{x})$ for $N=8, N=10$ and series solution presented by Wazwaz [7] for example 5 .

Here,

$$
y(x)=C^{T} \phi(x) \text { and } f(x, y)=\sin (y) .
$$

Expansion of $f(x, y)$ according to Taylor series is

$$
f(x, y)=\sin (y)=y-\frac{y^{3}}{6}+\frac{y^{5}}{120}+\cdots
$$

By considering only first three terms, $f(x, y)$ can be expressed as

$$
f(x, y)=C^{T} \phi(x)-\frac{\left(C^{T} \phi(x)\right)^{3}}{6}+\frac{\left(C^{T} \phi(x)\right)^{5}}{120}
$$

The coefficient vector $C^{T}$ is solved for $N=8 \& N=10$ and shown in Tables 4 and 5 respectively. The obtained results are presented in Fig. 7 in range [0,1]. Table 3 presents the comparison of solution $y(x)$ and absolute error with Wazwaz et al. [7], Pandey et al. [22] and Saadatmandi and Dehghan [29].

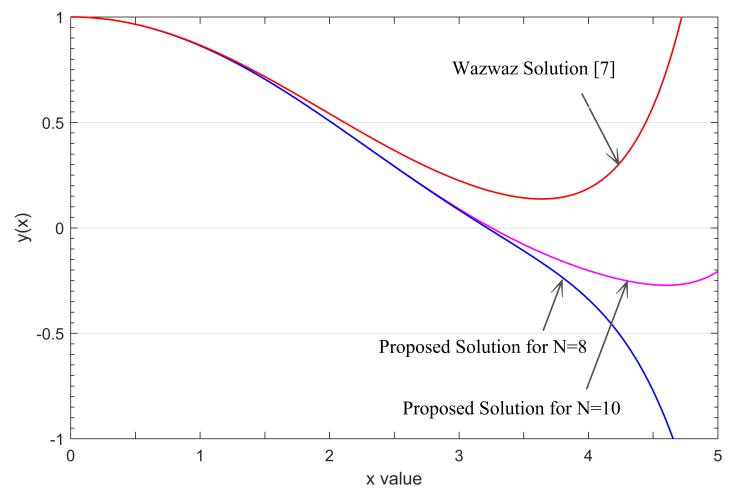

Fig. 7: Plot of obtained solution y(x) for $N=8, N=10$ and series solution presented by Wazwaz [7] for example 6 .

\section{Conclusions}

In this manuscript, we have proposed a modified approach to solve nonlinear Lane-Emden equation using first kind chebyshev operational matrix of differentiation. The benefit of this approach over other existing methods is that only operation matrix of differentiation have need of present the solution more precisely. This differentiation matrix contains mostly number of zeros, which reduces the run time and provides simplicity in computation. This algorithm generates nonlinear system of equations, which can be performed on computer using a program run in MATLAB2016. $N$ must be selected large sufficient to solve the Lane-Emden equation most precisely.

Consequently, the numerical solution concurs with the exact and series solution even with a small number of first kind of chebyshev polynomial used in estimation. Several examples have been worked out to reveal the accuracy and performance of modified algorithm than other recently developed methods.

\section{References}

[1] S. Chandrasekhar, Introduction to the study of stellar structure, Dover, New York, 1967.

[2] P.L. Chambre, On the solution of the Poission-Boltzmann equation with application to the theory of thermal explosions, J.Chem. Phys. 20(11) (1952) 1795-1797.

[3] O.U. Richardson, The Emission of electricity from Hot Bodies, Longman Green and Co., London, New York, 1921.

[4] J.H. Lane, On the theoritical tempreature of the sun under the hypothesis of a gaseous mass maintaining its volume by its internal heat and depending on the laws of gases known to terrestrial experiment, Am. J. Sci. Arts 2nd Ser. 50 (1870) 5774.

[5] R. Emden Gaskugeln, Anwendungen der mechanischen Warmen-theorie auf kosmologie and meteorologische problem, Leipzig, Teubner 1907.

[6] H.T. Davis, Introduction to Nonlinear Differential and integral Equations, Dover, New York, 1962.

[7] A.M. Wazwaz, A new algorithm for solving differential equations of Lane-Emden type, Appl. Math. Comput. 118 (2001) 287-310.

[8] C.M. Bender, K.A. Milton, S.S. Pinsky, L.M. Simmons Jr., A new perturbative approach to nonlinear problems, J. Math. Phys. 30 (1989) 1447-1455.

[9] H. Aminikhah and S. Kazemi, On the Numerical solution of singular Lane-Emden type equations using Cubic B-spline approximation, Int. J. Appl. Comput. Math

[10] R. Krivec and V.B. Mandelzweig, Numerical investigation of quasilinearization method in quantum mechanics, Comput. phys. commun. 138 (2001) 69-79. 
[11] R. Krivec and V.B. Mandelzweig, quasilinearization approach to computation with singular potentials, Comput. phys. commun. 179 (2008) 865-867.

[12] J.I. Ramos, linearization methods in classical and quantum mechanics, Comput. phys. commun. 153 (2003) 199-208.

[13] M. Dehgan and F. Shakeri, Approximation solution of a differential equation arising in astrophysics using the variational iteration method, New Astron. 13 (2008) 53-59.

[14] N.T. Shawagfeh, Nonperturbative approximate solution for Lane-Emden equation, J. Math. Phys. 34 (1993) 43-64.

[15] S.K. Vanani and A. Aminataei, On the numerical solution of differential equations of Lane-Emden type, Comput. Math. appl. 59 (2010) 2815-2820.

[16] R.D. Russell and L.F. Shampine, Numerical methods for singular boundary value problems, SIAM J. Numer Anal. 12 (1975) 13-36.

[17] Y. öztürk, M. Gülsu, An operational matrix equation for solving Lane-Emden equations arising in astrophysics, Math. Methods Appl. Sci. 37(15) (2014) 2227-2235.

[18] Y.H. Youssri, A new operational matrix of Caputo fractional derivatives of Fermat polynomials: an application for solving the Bagley-Torvik equation, Adv. Differ. Eqs. 73 (2017).

[19] W.M. Abd-Elhameed, Y.H. Youssri, Generalized Lucas polynomial sequence approach for fractional differential equations, Nonlinear Dyn. (2017)

[20] W.M. Abd-Elhameed, Y.H. Youssri, Fifth-kind orthonormal Chebyshev polynomial solutions for fractional differential equations, Comp. Appl. Math. (2017).

[21] K. Pranad, M. Dehgan, A.R. Rezaei, S.M. Ghaderi, An approximate algorithm for the solution of the nonlinear Lane-Emden type equations arising in astrophysics using Hermite function collocation method, Comput. Phys. Commun. 181 (2010) 1096-1108.
[22] R.K. Pandey, N. Kumar, A. Bhardwaj, G. Dutta, Solution of Lane-Emden type equations using Legendre operational matrix of differentiation, Appl. Math. Comput. 218 (2012) 7629 . 7637.

[23] E.H. Doha, W.M. Abd-Elhameed, Y.H. Youssri, Second kind Chebyshev operational matrix algorithm for solving differential equations of Lane-Emden type, New Astronomy 23-24 (2013) 113-117.

[24] W.M. Abd-Elhameed, New Galerkin operational matrix of derivatives for solving Lane-Emden singular-type equations, The Eur. Phys. J. Plus, 130(52) (2015).

[25] W.M. Abd-Elhameed, E.H. Doha, A.S. Saad, M.A. Bassuony, New galerkin operational matrices for solving lane-Emden type equations, Revista mexicana de astronomia y astrofisica, 52(1) (2016).

[26] S.A. Yousefi, Legendre wavelets method for solving differential equations of LaneEmden type, Appl. math. Comput. 181 (2006) 1417-1422.

[27] Y.H. Youssri, W.M. Abd-Elhameed, E.H. Doha, ultraspherical wavelets method for solving Lane-Emden type equations, Romanian J. Phys. (2015).

[28] M.H. Atabakzadeh, M.H.Akrami, G.H.Erjaee, Chebyshev operational matrix method for solving multi-order fractional ordinary differential equations, Appl. Math. Modelling 37(20-21) (2013) 8903-8911.

[29] A. Saadatmandi and M. Dehgan, A new operational matrix for solving fractional order differential equations, Comput. Math. Appl. 59 (2010) 1326-1336.

[30] C. Canuto, M.Y. Hussaini, A. Quarteoni, T.A. Zang, Spectral methods in fluid Dynamics, Springer, New York, 1988.

[31] A.W. Naylor, G.R. Sell, Linear operator theory in engineering and science, Applied Mathematical Sciences, second ed., vol. 40, Springer-Verlag, NewYork, Berlin, 1982. 